PROCEEDINGS OF THE

AMERICAN MATHEMATICAL SOCIETY

Volume 94 , Number 3, July 1985

\title{
EXTREME POINTS IN DUALS OF COMPLEX OPERATOR SPACES
}

\author{
ÅSVALD LIMA AND GUNNAR OLSEN
}

\begin{abstract}
We show that if $X$ and $Y$ are complex Banach spaces, and $K(X, Y)$ is the space of compact linear operators from $X$ into $Y$, then ext $B\left(K(X, Y)^{*}\right)=$ ext $B\left(X^{* *}\right) \otimes$ ext $B\left(Y^{*}\right)$.
\end{abstract}

Introduction. We shall let $X$ and $Y$ be complex Banach spaces and we denote the space of compact linear operators from $X$ into $Y$ by $K(X, Y)$. The space of linear bounded operators is denoted $L(X, Y) . B(X)$ is the closed unit ball of $X$ and $X^{*}$ is the dual space of $X$. ext $B(X)$ denotes the set of extreme points in $B(X)$.

For $x^{* *} \in X^{* *}$ and $y^{*} \in Y^{*}, x^{* *} \otimes y^{*} \in K(X, Y)^{*}$ is defined by

$$
x^{* *} \otimes y^{*}(T)=x^{* *}\left(T^{*} y^{*}\right) \text {. }
$$

In [3, Corollary 2], Fakhouri proved that for real spaces

$$
\text { ext } B\left(K(X, Y)^{*}\right) \subseteq \text { ext } B\left(X^{* *}\right) \otimes \operatorname{ext} B\left(Y^{*}\right) \text {. }
$$

A generalization of this result was given by Collins and Ruess in [2, Theorem 2.2]. The proofs given in [ 2 and 3] extend to complex spaces. Ruess and Stegall proved in [8, Theorem 1.3] the converse inclusion. Thus

$$
\text { ext } B\left(K(X, Y)^{*}\right)=\operatorname{ext} B\left(X^{* *}\right) \otimes \operatorname{ext} B\left(Y^{*}\right) \text {. }
$$

The proof in [8] is valid in the real case only. The object of this short note is to give a proof of the inclusion $\supseteq$ in $(*)$ for the complex case. The main result is

THEOREM 1. For real and complex Banach spaces, we have

$$
\text { ext } B\left(K(X, Y)^{*}\right)=\operatorname{ext} B\left(X^{* *}\right) \otimes \operatorname{ext} B\left(Y^{*}\right) \text {. }
$$

Proof of the main Result. We shall need Lemma 2 to test if $x^{* *} \otimes y^{*}$ is an extreme point.

Lemma 2. Let $\Phi: X \rightarrow C(K)$ be a linear isometry and let $x_{0}^{*} \in B\left(X^{*}\right)$ with $\left\|x_{0}^{*}\right\|=1$. Then $x_{0}^{*} \in \operatorname{ext} B\left(X^{*}\right)$ if and only if

$$
\left\{\begin{array}{l}
K_{0}=\left\{k \in K: \exists \lambda,|\lambda|=1, \Phi^{*}\left(\partial_{k}\right)=\lambda x_{0}^{*}\right\} \neq \varnothing \text { and if } \\
\|\mu\|=1=\left\|\Phi^{*} \mu\right\| \text { with } \Phi^{*} \mu \in \operatorname{span}\left\{x_{0}^{*}\right\}, \text { then } \\
\text { support } \mu \subseteq K_{0} .
\end{array}\right.
$$

Received by the editors May 28, 1984 and, in revised form, September 18, 1984.

1980 Mathematics Subject Classification. Primary 46B20; Secondary 47B05.

Key words and phrases. Compact operators, extreme points. 
Proof. Assume (\#) is satisfied. Suppose $x_{1}^{*}, x_{2}^{*} \in B\left(X^{*}\right)$ with $2 x_{0}^{*}=x_{1}^{*}+x_{2}^{*}$. Choose $\mu_{i} \in C(K)^{*}$ such that $\left\|\mu_{i}\right\|=\left\|x_{i}^{*}\right\|$ and $x_{i}^{*}=\Phi^{*} \mu_{i}$ for $i=1,2$. Let $\nu=\mu_{1}+$ $\mu_{2}$. Then we have $1=\|\nu\|=\left\|\Phi^{*} \nu\right\|$ and $\Phi^{*} \nu=x_{0}^{*}$. By (\#), $\nu$ is supported by $K_{0}$. Since

$$
1=\left\|x_{0}^{*}\right\| \leqslant\|\nu\|=|\nu|\left(K_{0}\right) \leqslant \frac{1}{2}\left(\left|\mu_{1}\right|+\left|\mu_{2}\right|\right)\left(K_{0}\right) \leqslant 1,
$$

$\mu_{1}$ and $\mu_{2}$ are supported by $K_{0}$ also. Hence $x_{i}^{*}=\Phi^{*} \mu_{i}=\lambda_{i} x_{0}^{*}$ for some $\left|\lambda_{i}\right| \leqslant 1$. This is clearly true if $\mu_{i}$ is a discrete measure. If $\mu_{i}$ is not discrete, then we use that we can approximate $\mu_{i}$ in the $w^{*}$-topology by discrete measures supported by $K_{0}$ and that $\Phi^{*}$ is $w^{*}$-continuous.

Thus

$$
2 x_{0}^{*}=x_{1}^{*}+x_{2}^{*}=\lambda_{1} x_{0}^{*}+\lambda_{2} x_{0}^{*}
$$

with $\left|\lambda_{i}\right| \leqslant 1$ and it follows that $\lambda_{1}=\lambda_{2}=1$. Hence $x_{0}^{*}$ is an extreme point.

The converse implication is simple and we shall not give the details.

It is well known that in a compact convex set, the only probability measure representing an extreme point is the point measure with unit mass [1, Corollary I.2.4]. There can be many more complex representing measures but, as the next lemma shows, their support is on a small set.

Lemma 3. Assume $X$ is a complex Banach space. Let $x_{0}^{*} \in$ ext $B\left(X^{*}\right)$ and let $\lambda$ be a measure on $B\left(X^{*}\right)$ with $\|\lambda\| \leqslant 1$ such that

$$
x_{0}^{*}(x)=\int x^{*}(x) d \lambda\left(x^{*}\right) \text { for all } x \in X .
$$

Then support $\mu \subseteq\left\{\alpha x_{0}^{*}:|\alpha|=1\right\}$.

Proof. There is a measurable function $\Phi$ on $B\left(X^{*}\right)$ such that $|\Phi|=1$ a.e. $|\lambda|$ and $\lambda=\Phi|\lambda|$. Define $\omega: B\left(X^{*}\right) \rightarrow B\left(X^{*}\right)$ by $\omega\left(x^{*}\right)=\Phi\left(x^{*}\right) x^{*}$. $\omega$ is measurable. We define a new measure $\omega(|\lambda|)$ on $B\left(X^{*}\right)$ by

$$
\omega(|\lambda|)(f)=\int f\left(\omega\left(x^{*}\right)\right) d|\lambda|\left(x^{*}\right)=\int f\left(\Phi\left(x^{*}\right) x^{*}\right) d|\lambda|\left(x^{*}\right) .
$$

For $x \in X$, we get

$$
\begin{aligned}
\omega(|\lambda|)(x) & =\int x\left(\Phi\left(x^{*}\right) x^{*}\right) d|\lambda|\left(x^{*}\right)=\int \Phi\left(x^{*}\right) x^{*}(x) d|\lambda|\left(x^{*}\right) \\
& =\int x^{*}(x) d \lambda\left(x^{*}\right)=x_{0}^{*}(x) .
\end{aligned}
$$

Thus $\omega(|\lambda|)$ is a probability measure representing $x_{0}^{*} \in \operatorname{ext} B\left(X^{*}\right)$. By Corollary I.2.4 in [1], we get $\omega(|\lambda|)=\partial_{x_{0}^{*}}$. Suppose $V$ is a compact subset in $B\left(X^{*}\right)$ with $|\lambda|(V)>0$ and $V \cap\left\{\alpha x_{0}^{*}:|\alpha|=1\right\}=\varnothing$. Let $\lambda_{1}=\chi_{V} \lambda$ and $\lambda_{2}=\lambda-\lambda_{1}$. Then we have $\lambda_{1} \perp \lambda_{2}$ and $\|\lambda\|=\left\|\lambda_{1}\right\|+\left\|\lambda_{2}\right\|$. By Corollary 3 in [7], we get

$$
\delta_{x_{0}^{*}}=\omega(|\lambda|)=\omega\left(\left|\lambda_{1}\right|\right)+\omega\left(\left|\lambda_{2}\right|\right) \text {. }
$$


If $f \geqslant 0$ is a continuous function on $B\left(X^{*}\right)$ with $f=0$ on $\left\{\alpha x_{0}^{*}:|\alpha|=1\right\}$ and $f=1$ on $\{\alpha v:|\alpha|=1, v \in V\}$, then we have

$$
\begin{aligned}
0 & =\partial_{x_{0}^{*}}(f) \geqslant \omega\left(\left|\lambda_{1}\right|\right)(f) \\
& =\int f\left(\Phi\left(x^{*}\right) x^{*}\right) \chi_{V}\left(x^{*}\right) d|\lambda|\left(x^{*}\right) \geqslant|\lambda|(V)>0 .
\end{aligned}
$$

This contradiction shows that support $|\lambda| \subseteq\left\{\alpha x_{0}^{*}:|\alpha|=1\right\}$.

Before we give the proof of Theorem 1, we shall give the complex version of Lemma 1.5 in [8].

Lemma 4. Assume $x_{0}^{*} \in \operatorname{ext} B\left(X^{*}\right)$ and that $\mu$ is a positive measure on $B\left(X^{*}\right)$ with $\|\mu\| \leqslant 1$. If $\left|x_{0}^{*}(x)\right| \leqslant \int\left|x^{*}(x)\right| d \mu\left(x^{*}\right)$ for all $x \in X$, then support $\mu \subseteq\left\{\alpha x_{0}^{*}:|\alpha|=\right.$ $1\}$.

Proof. As in the proof of Lemma 1.5 in [8], we find $h \in L^{\infty}\left(B\left(X^{*}\right), \mu\right)$ such that

$$
x_{0}^{*}(x)=\int x^{*}(x) h\left(x^{*}\right) d \mu\left(x^{*}\right) \text { for all } x \in X .
$$

We have $\|h d \mu\| \leqslant 1$ and by Lemma 3 it follows that $h d \mu$ has support in $\left\{\alpha x_{0}^{*}\right.$ : $|\alpha|=1\}$. Since necessarily, $1=\|\mu\|=\|h d \mu\|$, it follows that support $\mu \subseteq\left\{\alpha x_{0}^{*}\right.$ : $|\alpha|=1\}$.

Proof of Theorem 1. Let $x_{0}^{* *} \in \operatorname{ext} B\left(X^{* *}\right)$ and $y_{0}^{*} \in \operatorname{ext} B\left(Y^{*}\right)$ and let $h^{*}=$ $x_{0}^{* *} \otimes y_{0}^{*} \in K(X, Y)^{*}$. Let $K=B\left(X^{* *}\right) \times B\left(Y^{*}\right)$ with product $w^{*}$-topology. Let $\Phi$ : $K(X, Y) \rightarrow C(K)$ be defined by

$$
\Phi(T)\left(x^{* *} ; y^{*}\right)=x^{* *}\left(T^{*} y^{*}\right) .
$$

$\Phi$ is a linear isometry. Let $K_{0}=\left\{k \in K: \exists \lambda,|\lambda|=1, \Phi^{*}\left(\delta_{k}\right)=\lambda h^{*}\right\}$ and let $Z_{0}=\left\{\left(\alpha x_{0}^{* *}, \beta y_{0}^{*}\right):|\alpha|=|\beta|=1\right\}$. Then clearly $Z_{0} \subseteq K_{0} \subseteq K$.

We shall use Lemma 2 to show that $h^{*}$ is an extreme point. Suppose $\mu$ is a measure on $K$ with $\|\mu\|=1=\left\|\Phi^{*} \mu\right\|$ and assume $\Phi^{*} \mu \in \operatorname{span}\left\{h^{*}\right\}$. Then $\Phi^{*} \mu=\lambda h^{*}$ and we may assume $\lambda=1$.

Let $x^{*} \in X^{*}$ and $y \in Y$. Define $T \in K(X, Y)$ by $T(x)=x^{*}(x) y$. Then we get

$$
\begin{aligned}
x_{0}^{* *}\left(x^{*}\right) y_{0}^{*}(y) & =h^{*}(T)=\Phi^{*} \mu(T)=\mu(\Phi(T)) \\
& =\int_{K} \Phi(T)\left(x^{* *}, y^{*}\right) d \mu\left(x^{* *}, y^{*}\right) \\
& =\int_{K} x^{* *}\left(x^{*}\right) y^{*}(y) d \mu\left(x^{* *}, y^{*}\right) .
\end{aligned}
$$

Let $\mu_{1}=\mid \mu \|_{B\left(X^{* *}\right)}$ and $\mu_{2}=\mid \mu \|_{B\left(Y^{*}\right)}$. Then we get

$$
\left|x_{0}^{* *}\left(x^{*}\right) y_{0}^{*}(y)\right| \leqslant \int_{B\left(X^{* *}\right)}\left|x^{* *}\left(x^{*}\right)\right| d \mu_{1}\left(x^{* *}\right) .
$$

Thus it follows that

$$
\left|x_{0}^{* *}\left(x^{*}\right)\right| \leqslant \int_{B\left(X^{* *}\right)}\left|x^{* *}\left(x^{*}\right)\right| d \mu_{1}\left(x^{* *}\right) .
$$


By Lemma 4, support $\mu_{1} \subseteq\left\{\alpha x_{0}^{* *}:|\alpha|=1\right\}$. Similarly, support $\mu_{2} \subseteq\left\{\beta y_{0}^{*}:|\beta|=1\right\}$. Hence support $\mu \subseteq Z_{0} \subseteq K_{0}$. By Lemma $2, h^{*}$ is an extreme point in $B\left(K(X, Y)^{*}\right)$.

Applications. Using Theorem 1, the proof of Theorem 5.6 in [5] extends to the complex case.

THEOREM 5. Let $X$ and $Y$ be real or complex Banach spaces. If $K(X, Y)$ contains a proper $M$-summand, then $Y$ contains a proper $M$-summand or $X$ contains a proper L-summand.

For reflexive spaces we get

COROllary 6. Assume $X$ is reflexive. If $K(X)$ contains a proper $M$-ideal, then $X$ or $X^{*}$ contains a proper $M$-summand.

Corollary 7. For $1<p<\infty, K\left(l_{p}\right)$ contains no proper $M$-ideal.

It is well known that the $M$-ideals coincide with the closed two-sided ideals in $C^{*}$-algebras. Thus Corollary 7 gives as a special case the well-known fact that $K\left(l_{2}\right)$ contains no proper closed two-sided ideals. Corollary 7 was first proved by Smith and Ward [9] and Flinn [4]. Corollary 6 extends their result to a much larger class of spaces.

From the proof of Theorem 5.7 in [6], it follows that if $T \in L(X, Y)$ and $\varepsilon>0$, then there exists $S \in L(X, Y), y_{0}^{*} \in \operatorname{ext} B\left(Y^{*}\right)$ and $x_{0}^{* *} \in \operatorname{ext} B\left(X^{* *}\right)$ such that $\|S-T\|<\varepsilon$ and $\|S\|=x_{0}^{* *}\left(S^{*} y_{0}^{*}\right)$. For $h=x_{0}^{* *} \otimes y_{0}^{*} \in \operatorname{ext} B\left(K(X, Y)^{*}\right)$, let $\hat{h}$ denote the natural extension to $L(X, Y)$ defined by $\hat{h}(T)=x_{0}^{* *}\left(T^{*} y_{0}^{*}\right)$. Thus we have that the convex hull of $\left\{\hat{h}: h \in \operatorname{ext} B\left(K(X, Y)^{*}\right)\right\}$ is $w^{*}$-dense in $B\left(L(X, Y)^{*}\right)$.

ACKNOWLEDGEMENT. The authors thank the referee for his valuable suggestions. The idea to use Lemma 2 is due to him.

\section{REFERENCES}

1. E. Alfsen, Compact convex sets and boundary integrals, Ergebnisse Math. Grenzgebiete, Bd. 57, Springer-Verlag, Berlin and New York, 1971.

2. H. S. Collins and W. Ruess, Weak compactness in space of compact operators and of vector-valued functions, Pacific J. Math. 106 (1983), 45-71.

3. H. Fakhouri, Approximation par des opérateurs compacts ou faiblement compacts à valeurs dans $C(X)$, Ann. Inst. Fourier (Grenoble) 27 (1977), 147-167.

4. P. Flinn, $A$ characterization of $M$-ideals in $B\left(l_{p}\right)$ for $1<p<\infty$, Pacific J. Math. 98 (1982), 73-80.

5. P. Harmand and $\AA$. Lima, Banach spaces which are $M$-ideals in their biduals, Trans. Amer. Math. Soc. 283 (1984), 253-264.

6. A. Lima, Intersection properties of balls in spaces of compact operators, Ann. Inst. Fourier (Grenoble) 28 (1978), 35-65.

7. G. Olsen, On the classification of complex Lindenstrauss spaces, Math. Scand. 35 (1974), 237-258.

8. W. M. Ruess and C. P. Stegall, Extreme points in duals of operator spaces, Math. Ann. 261 (1982), 535-546.

9. R. R. Smith and J. D. Ward, M-ideal structure in Banach algebras, J. Funct. Anal. 27 (1978), $337-349$.

Department of Mathematics and Statistics, Norwegian Agricultural University, 1432 AAS - NLH, NORWAY 